\title{
Multigrid and Defect Correction for the Efficient Solution of the Steady Euler Equations
}

\author{
Barry Koren, Stefan Spekreijse \\ Centre for Mathematics and Computer Science \\ P.O. Box 4079, 1009 AB Amsterdam. The Netherlands
}

\begin{abstract}
An efficient iterative solution method for second-order accurate discretizations of the $2 D$ Steady Euler equations is described and results are shown. The method is based on a nonlinear multigrid method and on the defect correction principle. Both first- and second-order accurate finite-volume upwind discretizations are considered. In the second-order discretization a limiter is used.

An Iterative Defect Correction process is used to approximately solve the system of second-order discretized equations. In each iteration of this process, a solution is computed of the first-order system with an appropriate right-hand side. This solution is computed by a nonlinear multigrid method, where Symmetric Gauss-Seidel relaxation is used as the smoothing procedure.

The computational method does not require any tuning of parameters. Flow solutions are presented for an airfoil and a bi-airfoil with propeller disk. The solutions show good resolution of all flow phenomena and are obtained at low computational cost. Particularly with respect to efficiency, the method contributes to the state of the art in computing steady Euler flows with discontinuities.
\end{abstract}

1980 Mathematics Subject Classification: 35L65, 35L67, 65N30, 76G15, 76H05.

Key Words and Phrases: steady Euler equations, multigrid methods, defect correction.

Note: This work was supported in part by the Netherlands Technology Foundation (STW)

\section{INTRODUCTION}

The Euler equations describe compressible inviscid gas flows with rotation. They are widely used in the aerospace industry. The Euler equations are derived by considering the laws of conservation of mass, momentum and energy for an inviscid gas. The result is a nonlinear hyperbolic system of conservation laws. Only for very simple flow problems, analytical solutions exist. For almost all engineering problems solutions must be found numerically. Several discretization methods have been developed which yield solutions of good quality (good resolution of shock waves, slip lines, etc.). However, generally the computational cost is high. In 1983 a project was started at the Centre for Mathematics and Computer Science (CWI) in Amsterdam for the development of more efficient methods. So far, a multigrid method for the solution of the 2D steady Euler equations has been developed, implemented and tested.

In the method, the steady Euler equations are discretized by a finite-volume upwind discretization [9]. Both first- and second-order discretizations are obtained by the projection-evolution approach [14]. In the projection-stage of this approach the discrete values, located in the volume centers, are interpolated to yield continuous distributions in each volume. First-order accuracy is obtained by piecewise constant interpolation, second-order accuracy by piecewise linear interpolation. In case of flows with discontinuities (shock waves or slip lines), the occurrence of spurious non-monotonicity (wiggles) when using a second-order interpolation, is suppressed by the use of a limiter in the interpolation formulae [23]. In this paper we use the Van Albada limiter [1,20]. In the evolution-stage, a Riemann problem is considered for the computation of the flux at each volume wall. To approximately solve each Riemann problem we use the Osher scheme [16].

To obtain solutions of the system of tirst-order discretized equations, the nested nonlinear multigrid (FMG-FAS-) iteration method appears to be a very efficient solution method [9]. However, the multigrid solution of a system of second-order discretized equations appears to be less efficient [21]. 
problems) and the central scheme. A disadvantage of these $\kappa$-schemes is that near discontinuities, spurious non-monotonicity (wiggles) appears [11]. A way to avoid this is by using a limiter. We modify the $\kappa$-schemes by introducing a limiter such that the schemes become monotone and remain second-order accurate. Let $q_{i+1 / 2, j}^{l}(k)$ and $q_{i+1 / 2, j}^{r(k)}$ be the $k$ th component $(k=1,2,3,4)$ of $q_{i+1 / 2, j}^{l}$ and $q_{i+1 / 2, j}^{r}$. We rewrite $(2.10)$ as

$$
\begin{aligned}
& q_{i+1 /, j}^{l(k)}=q_{i, j}^{(k)}+1 / 2 \psi_{k}\left(R_{i, j}^{(k)}\right)\left(q_{i, j}^{(k)}-q_{i-1, j}^{(k)}\right), \text { and } \\
& q_{i+1 / 2, j}^{r(k)}=q_{i+1, j}^{(k)}+1 / 2 \psi_{k}\left(1 / R_{i+1, j}^{(k)}\right)\left(q_{i+1, j}^{(k)}-q_{i+2, j}^{(k)}\right),
\end{aligned}
$$

where

$$
R_{i, j}^{(k)}=\frac{q_{i+1, j}^{(k)}-q_{i, j}^{(k)}}{q_{i, j}^{(k)}-q_{i-1, j}^{(k)}},
$$

and where $\psi_{k}: \mathbb{R} \rightarrow \mathbb{R}$ is defined by

$$
\psi_{\kappa}(R)=\frac{1-\kappa}{2}+\frac{1+\kappa}{2} R .
$$

If we replace $\psi_{\kappa}(R)$ in $(2.11)$ by $\psi_{\kappa}^{\lim }(R)$, where $\psi_{\kappa}^{\lim }(R)$ is defined by

$$
\psi_{\kappa}^{\lim }(R)=\frac{2 R}{R^{2}+1} \psi_{\kappa}(R)
$$

then (2.11) results in a monotone and yet second-order accurate scheme [20]. The function $\psi_{k}^{\lim }: \mathbb{R} \rightarrow \mathbb{R}$ is called the limiter. The choice $\kappa=0$ corresponds with the Van Albada limiter [1,20], hence the Van Albada limiter can be considered as a modification of the Fromm scheme. Notice that we have a piecewise linear interpolation in each volume if

$$
q_{i+1 / 2, j}^{l}-q_{i, j}=q_{i, j}-q_{i-1 / 2, j}^{r}
$$

It can be easily seen that (2.15) holds if

$$
\psi_{\kappa}^{\lim }(R)=R \psi_{\kappa}^{\lim }(1 / R)
$$

and this is only true for $\kappa=0$. An advantage of the Van Albada limiter is that in the neighbourhood of discontinuities the scheme resembles the fully one-sided upwind scheme, which is a natural scheme in such regions. For all flow solutions presented in this paper we used $\psi_{0}^{\lim }(R)$ although $\psi_{1 / 3}^{\lim }(R)$ seems a reasonable choice as well.

In case $\Omega_{i, j}$ is a boundary volume, so that for example $2 \Omega_{i+1 / 2, j}$ is part of the domain boundary, no limiter can be used to compute $q_{i+1 / 2, j}^{l}$ and $q_{i-1 / 2, j}^{r}$. In this case we use a simple linear interpolation, i.e.

$$
\begin{aligned}
& q_{i+1 / 2, j}^{l}=q_{i, j}+1 / 2\left(q_{i, j}-q_{i-1, j}\right), \text { and } \\
& q_{i-1 / 2, j}^{r}=q_{i, j}-1 / 2\left(q_{i, j}-q_{i-1, j}\right) .
\end{aligned}
$$

The boundary conditions, together with the state $q_{i+1 / 2, j}^{l}$, are used to compute the state $q_{i+1 / 2, j}^{r}$. This computation is done by considering the Riemann initial-boundary value problem $[9,17]$. The flux $f_{i+1 / 2, j}$ at $\partial \Omega_{i+1 /, j}$ is computed by $(2.8)$.

\section{SOLUTION METHOD}

The method to solve the system of nonlinear discretized equations is based on a multigrid technique. For readers unfamiliar with multigrid techniques we refer to [3,5].

Let

$$
F_{h}^{1}\left(q_{h}\right)=r_{h},
$$

and 


$$
F_{h}^{2}\left(q_{h}\right)=r_{h}
$$

be first- and second-order accurate finite-volume upwind discretizations of the 2D steady Euler equations with source term $r$. Hence, $\left(F_{h}^{1}\left(q_{h}\right)\right)_{i, j}=F_{i, j}$ is defined by (2.7), (2.8) and (2.9), and $\left(F_{h}^{2}\left(q_{h}\right)\right)_{i, j}=F_{i, j}$ is defined by (2.7), (2.8), (2.11) and (2.12) with $\psi_{\kappa}(R)=\psi_{0}^{\text {lim }}(R)$ (the Van Albada limiter). Although in general $r=0$, we prefer to describe the solution method for systems with an arbitrary right-hand side. The subscript $h$ denotes the meshwidth. To apply multigrid we construct a nested set of grids, such that each volume in a grid is the union of 4 volumes in the next finer grid, in the obvious way. Let $\Omega_{h_{1}}$ with $h_{1}>h_{2}>\cdots>h_{l}=h$ be a sequence of such nested grids. So $\Omega_{h_{1}}$ and $\Omega_{h_{1}}$ are respectively the coarsest and the finest grid.

The solution method for (3.2) can be divided into three successive stages. The first stage is the Full Multigrid (FMG-) method, which is used to find a good initial approximation of (3.1). The second stage is a nonlinear multigrid (FAS-) iteration method, which is used to find a better approximate solution of (3.1). The first iterand is the solution obtained by the FMG-method. The third and last stage is an Iterative Defect Correction (IDeC-) process, which is used to find an approximate solution of (3.2). The first iterand of this process is obtained from the second stage. We will now discuss these stages more fully.

\section{Stage I: The Full Multigrid (FMG-) method.}

Let

$$
F_{h_{i}}^{l}\left(q_{h_{i}}\right)=r_{h_{i}}
$$

be the first-order discretization on $\Omega_{h_{1}}, i=1,2, \ldots, l$. The FMG-method (or nested iteration) starts with a crude initial exstimate of $q_{h_{1}}$; the solution on the coarsest grid. To obtain an initial estimate on the finer grid $\Omega_{h_{t+1}}$, first the solution on the next coarser grid $\Omega_{h_{h}}$ is improved by a single FAS-iteration (stage II). Hereafter this improved approximation is interpolated to the finer grid $\Omega_{h_{i+1}}$. These steps are repeated until the highest level has been reached. The interpolation used to obtain the first guess on a next finer grid is a bilinear interpolation. For this purpose the grid $\Omega_{h}$ is subdivided into disjunct sets of $2 \times 2$ volumes. The four states corresponding with each set are interpolated in a bilinear way, and since each volume of $\Omega_{h_{1}}$ overlaps $2 \times 2$ finer grid volumes of $\Omega_{h_{t+1}}, 4 \times 4$ new states are obtained on $\Omega_{h_{i+1}}$.

Stage II: The nonlinear multigrid (FAS-) iteration method.

To find a better approximation to (3.1) we apply the FAS-iteration method on the finest grid $\left(\Omega_{h_{1}}\right)$. One FAS-iteration on a general grid $\Omega_{h}$, is recursively defined by the following steps:

(0) Start with an approximate solution of $q_{h}$.

(1) Improve $q_{h_{1}}$ by application of $p$ (pre-) relaxation iterations to $F_{h_{1}}^{1}\left(q_{h_{1}}\right)=r_{h_{1}}$.

(2) Compute the defect $d_{h_{i}}:=r_{h_{i}}-F_{h}^{1}\left(q_{h_{1}}\right)$.

(3) Find an approximation of $q_{h_{t-1}}$ on the next coarser grid $\Omega_{h_{t-1}}$. Either use $q_{h_{t-1}}:=\hat{I}_{h_{t}}^{h_{t-1}} q_{h_{t}}$, where $\hat{I}_{h_{t}}^{h_{t-1}}$ is a restriction operator, or use the last obtained approximation $q_{h_{t-1}}$. (We use this last obtained approximation.)

(4) Compute $r_{h_{t-1}}:=F_{h_{t-1}}^{1}\left(q_{h_{t-1}}\right)+I_{h_{t}}^{h_{t-1}} d_{h_{i}}$ where $I_{h_{i}}^{h_{i-1}}$ is another restriction operator.

(5) Approximate the solution of $F_{h_{t-1}}^{1}\left(q_{h_{t-1}}\right)=r_{h_{t-1}}$ by $\sigma$ FAS- iterations on $\Omega_{h_{t-1}}$. The result is called $\tilde{q}_{h_{t-1}} \cdot(\sigma=1$ results in a so-called V-cycle and $\sigma=2$ in a W-cycle.)

(6) Correct the current solution by $q_{h_{t}}:=q_{h_{t}}+I_{h_{t-1}}^{h_{t}}\left(\tilde{q}_{h_{t-1}}-q_{h_{t-1}}\right)$, where $I_{h_{t-1}}^{h_{t}}$ is a prolongation operator.

(7) Improve $q_{h_{1}}$ by application of $q$ (post-) relaxation iterations to $F_{h_{i}}^{1}\left(q_{h_{i}}\right)=r_{h_{1}}$.

The steps (2) - (6) are called the coarse-grid correction. These steps are skipped on the coarsest grid. 
In order to complete the description of a FAS-iteration we have to discuss: (i) the choice of the transfer operators $I_{h_{i}}^{h_{1}}, I_{h_{h^{\prime}}}^{h^{\prime}}$ and $I_{h_{s}}^{h_{1}}$, (ii) the relaxation method, and (iii) the FAS-strategy, i.e. the numbers $p, q$ and $\sigma$.

(i) Choice of the operators:

The restriction operators $\hat{I}_{h_{+}}^{h_{1}}$ ' and $I_{h_{1}}^{h_{1}, 1}$ are defined by

$$
\begin{aligned}
& \left(q_{h_{i},}\right)_{h, j}=\left(\hat{I}_{h_{i}}^{h_{i}-1} q_{h_{i}}\right)_{i, j}:=\frac{1}{4}\left\{\left(q_{h_{i}}\right)_{2 i, 2 j}+\left(q_{h_{i}}\right)_{2 i-1,2 j}+\left(q_{h_{i}}\right)_{2 i, 2 j-1}+\left(q_{h_{i}}\right)_{2 i-1,2 j-1}\right\} \text {, and } \\
& \left(r_{h_{i},}\right)_{h, j}=\left(I_{h_{i}}^{h_{i}} ' r_{h_{i}}\right)_{i, j}:=\left(r_{h_{i}}\right)_{2 i, 2 j}+\left(r_{h_{i}}\right)_{2 i-1,2 j}+\left(r_{h_{1}}\right)_{2 i, 2 j-1}+\left(r_{h_{1}}\right)_{2 i-1,2 j-1} .
\end{aligned}
$$

The prolongation operator $I_{h_{i-1}^{\prime}}^{h_{1}}$ is defined by

$$
\left(I_{h_{1-1}}^{h_{i}} q_{h_{t-1}}\right)_{2 i, 2 j}=\left(I_{h_{i, 1}}^{h_{i}} q_{h_{t-1}}\right)_{2 t-1,2 j}=\left(I_{h_{t-1}}^{h_{i}} q_{h_{t-1}}\right)_{2 i, 2 j-1}=\left(I_{h_{t-1}}^{h_{i}} q_{h_{t-1}}\right)_{2 i-1,2 j-1}:=\left(q_{h_{i-1}}\right)_{i, j} \text {. }
$$

Note that this prolongation is different from the bilinear interpolation used in FMG. By defining the transfer operators in this way, it can be verified that

$$
F_{h_{t-1}}^{1}=I_{h_{t}}^{h_{i-1}} F_{h_{i}}^{1} I_{h_{t-1}}^{h_{1}} \text {, }
$$

i.e. the first-order coarse grid discretizations of the steady Euler equations are Galerkin approximations of the fine grid discretizations. This is a very important property because it implies that the coarse grid correction efficiently reduces the smooth component in the residual.

(ii) The relaxation method:

We use Collective Symmetric Gauss-Seidel (CSGS-) relaxation. Collective means that the four variables corresponding to a single volume are relaxed simultaneously. At each volume visited we solve the four nonlinear equations by Newton's method (local linearization). It appears that a single Newton iteration is sufficient. For details about the local linearization formulae we refer to [9].

(iii) The FAS-strategy:

We use a fixed strategy: $\sigma=1$ and $p=q=1$, i.e. we use V-cycles with one pre- and one postrelaxation.

Stage III : The Iterative Defect Correction (IDeC-) process.

For an introduction to the defect correction approach we refer to [2]. We approximate the solution of (3.2) with the IDeC-process:

$$
F_{h}^{1}\left(q_{h}^{(n+1)}\right)=F_{h}^{\mathrm{l}}\left(q_{h}^{(n)}\right)+\left(r_{h}-F_{h}^{2}\left(q_{h}^{(n)}\right)\right), n=0,1,2, \ldots,
$$

where $q_{h}^{(0)}$ is the solution obtained in stage II. It is clear that the fixed point of this iteration process is the solution of (3.2). In fact it is not really necessary to iterate until convergence. For smooth solutions a single IDeC-iteration is sufficient to obtain second-order accuracy [7]. For solutions with discontinuities experience shows that one or a few IDeC-iterations significantly improve the accuracy of the solution [11].

For each IDeC-iteration we have to solve a first-order system with an appropriate right-hand side. It appeared that it is inefficient to solve this system very accurately. Application of a single FASiteration to approximate $q_{h}^{(n+1)}$ in (3.8) usually is the most efficient strategy [7,11].

In fig. 3.1. we give an illustration of the complete solution process. Suppose there are 5 nested grids $(l=5)$. Between two succeeding points A,B we have one FAS-iteration (V-cycle). Between two succeeding points B,A we have a bilinear interpolation in the FMG-stage, and an appropriate righthand side computation in the IDeC-stage.

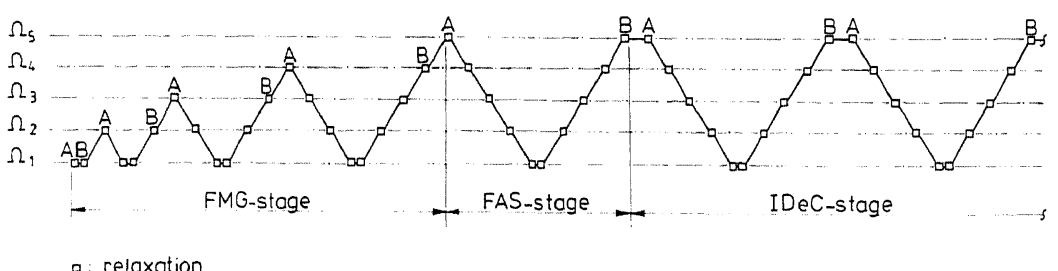

Fig. 3.1: Schematic representation of (a 5-level) solution process. 


\section{RESULTS}

To show that the method is feasible for a good and efficient computation of typical Euler flows, we consider two standard Euler test cases for a NACA0012-airfoil $\left(M_{\infty}=0.85, \alpha=1^{\circ}\right.$ and $M_{\infty}=1.2, \alpha=7^{\circ}$ ) and compare our results with results from [24]. ( $M_{\infty}$ denotes the Mach number at infinity and $\alpha$ the airfoil's angle of attack.) Both airfoil flows are isenthalpic, i.e. $(E+p) / \rho$ is uniformly constant. (This fact is not exploited in our computations.) To test our method in case of non-isenthalpic Euler flow we consider a configuration composed of two NACA0012-airfoils and a propeller disk. The NACA0012-airfoils are placed in parallel formation, the propeller disk is placed between the airfoil noses (fig. 4.1). The configuration can be interpreted as a model for a bi-plane with airscrew(s) between its leading edges. The propeller disk is modelled as a line-distribution of $x$ momentum and energy sources. For the NACA0012-bi-airfoil with propeller disk, no results to compare with are available.

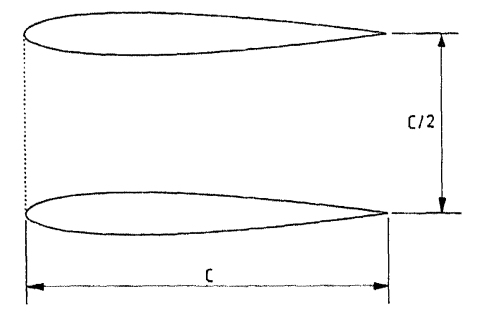

Fig. 4.1: NACA0012-bi-airfoil with propeller disk.

The NACA0012-airfoil:

For the NACA0012-airfoil we use a $128 \times 32$ O-type grid with the outer boundary at an approximate distance from the airfoil of 100 chord lengths (fig. 4.2). Following [8,11], we impose unperturbed flow conditions at the outer boundary, although we do not overimpose. For the subsonic outer boundary of the first test case we impose 3 conditions at the inflow part of that boundary $\left(u=M_{\infty} \cos \alpha, v=M_{\infty} \sin \alpha, c=1\right)$, and 1 condition at the outflow part $\left(u=M_{\infty} \cos \alpha\right)$. For the supersonic test case we impose 4 conditions at inflow $\left(u=M_{\infty} \cos \alpha, v=M_{\infty} \sin \alpha, c=1, p=1\right)$, and nothing at outflow. For both test cases we perform $10 \mathrm{IDeC}$-iterations and use a multigrid algorithm with 4 coarser grids.

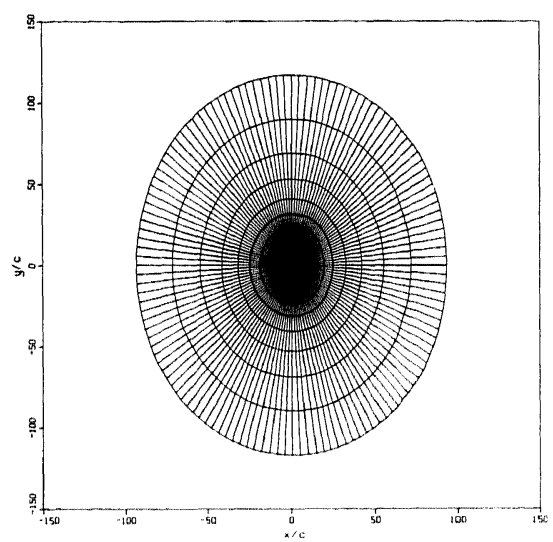

a. In full.

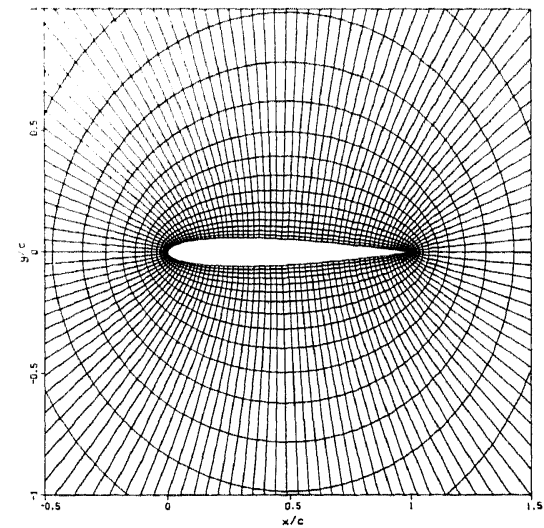

b. In detail.

Fig. 4.2: 128×32-grid NACA0012-airfoil. 


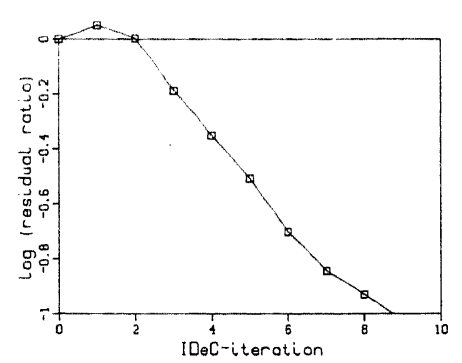

a. Convergence history residual ratio.

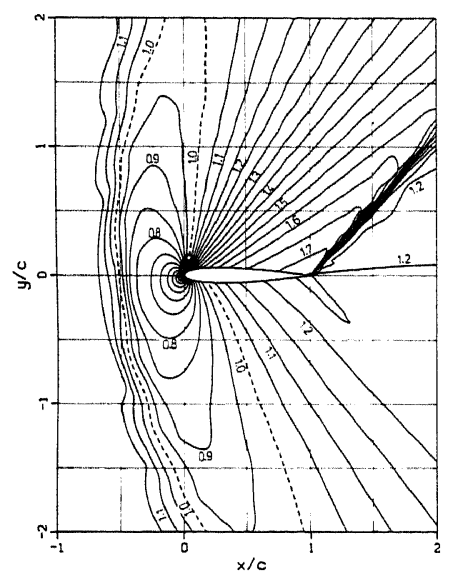

c. Mach number distributions; present result (left) and rusult Veuillot \& Vuillot (right).

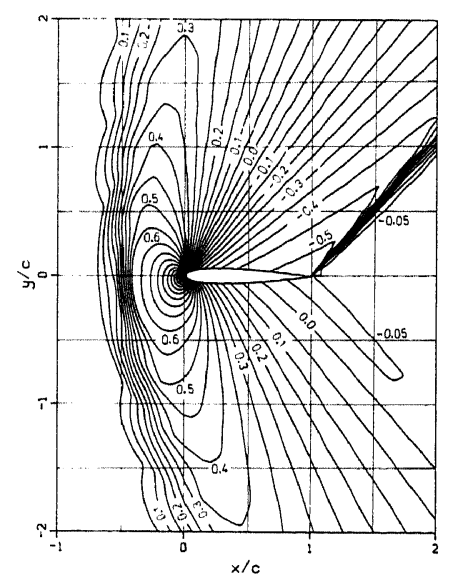

d. Present pressure distribution $\left(c_{p}\right)$.

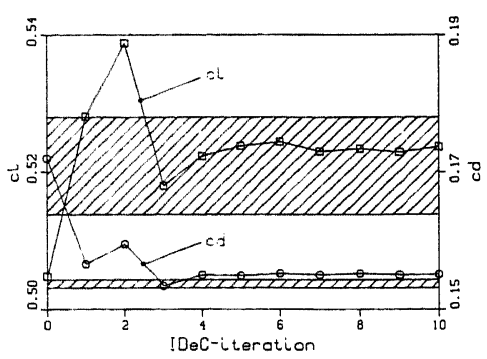

b. Convergence history lift and drag coefficient.

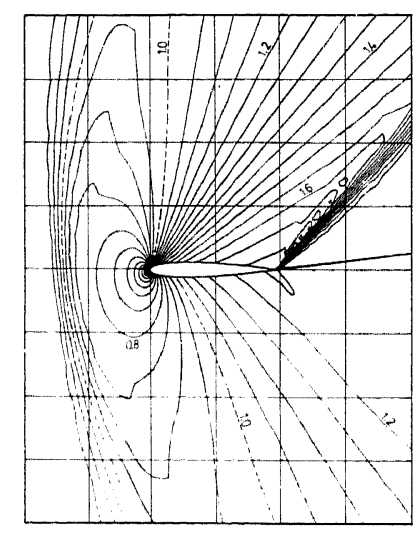

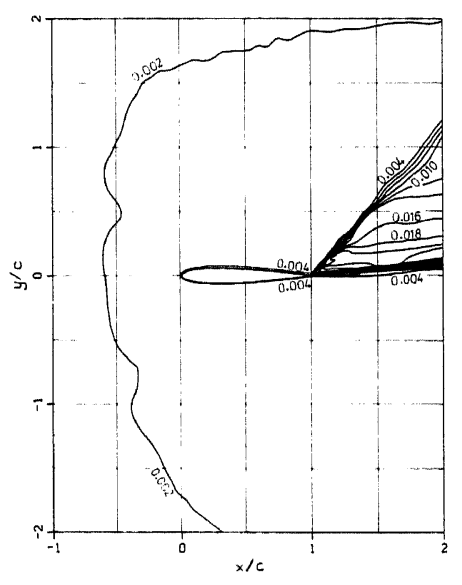

e. Present entropy distribution $\left(s / s_{\infty}-1\right)$.

Fig. 4.4: Results for NACA0012-airfoil at $M_{\infty}=1.2, \alpha=7^{\circ}$. 
The NACA0012-bi-airfoil with propeller disk:

For this configuration we use a $128 \times 48$-grid as shown in fig. 4.5. The non-smoothness of the grid at the airfoil noses possibly leads to a solution of worse quality. In [10], where only a first-order accurate discretization was used, we already observed a spurious entropy rise along a kinked wall. However, to investigate the capabilities of a second-order discretization with respect to this non-smooth grid, and simply to avoid extensive grid generation efforts we prefer the present grid to a smooth grid in a multi-domain approach. The outer boundary of the grid is taken at an approximate distance from the configuration of 10 chord lengths. For the solution algorithm 5 IDeC-iterations and 4 coarser grids are taken.

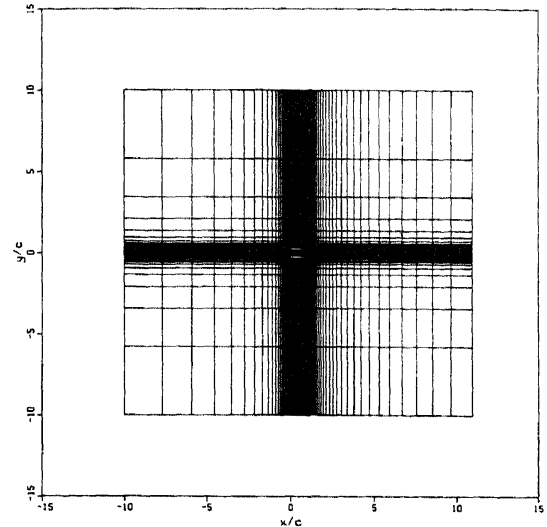

a. In full.

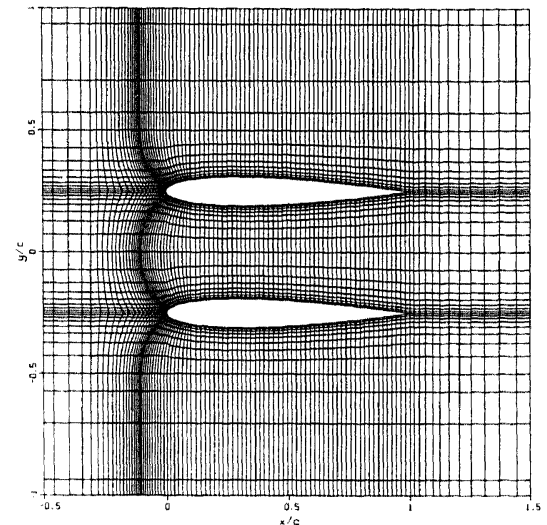

b. In detail.

Fig. 4.5: $128 \times 48$-grid NACA0012-bi-airfoil with propeller disk.

We consider the configuration twice at $M_{\infty}=0.5, \alpha=2^{\circ}$; namely with the propeller disk switched off and with the propeller disk switched on. In both cases we impose unperturbed conditions at the outer boundary $\left(u=M_{\infty} \cos \alpha, v=M_{\infty} \sin \alpha\right.$ and $c=1$ at inflow, and $p=1$ at outflow). We assume the propeller disk to be located inside the volumes, i.e. we assume that the propeller does not coincide with any volume wall. In each volume $\Omega_{i, j}$ which is intersected by the propeller disk, a local line source of $x$-momentum and energy per unit of length and time $\left(\left(\delta_{2}\right)_{i, j}\right.$ respectively $\left.\left(\delta_{4}\right)_{i, j}\right)$ are computed in the following way. We assume a sudden rise in the pressure:

$$
p=(1+\delta) p_{i, j},
$$

with $\delta$ constant. Further, following $[12$, ch.3] we assume the flow through the propeller disk to be isentropic:

$$
p \rho^{-\gamma}=p_{i, j} \rho_{i, j}^{-\gamma} \text {. }
$$

With these two relations and with the steady jump relations

$$
\begin{aligned}
& \rho u-\rho_{i, j} u_{i, j}=0, \\
& \left(\rho u^{2}+p\right)-\left(\rho_{i, j} u_{i, j}^{2}+p_{i, j}\right)=\left(\delta_{2}\right)_{i, j}, \\
& \rho u v-\rho_{i, j} u_{i, j} v_{i, j}=0, \\
& \left\{\frac{\gamma}{\gamma-1} p+1 / 2 \rho\left(u^{2}+v^{2}\right)\right\} u-\left\{\frac{\gamma}{\gamma-1} p_{i, j}+1 / 2 \rho_{i, j}\left(u_{i, j}^{2}+v_{i, j}^{2}\right)\right\} u_{i, j}=\left(\delta_{4}\right)_{i, j},
\end{aligned}
$$



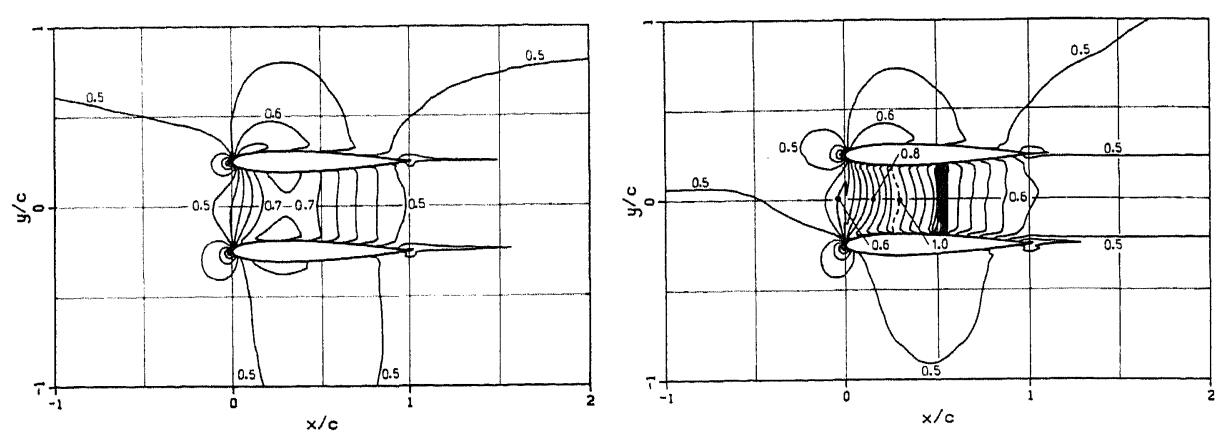

a. Mach number distributions.
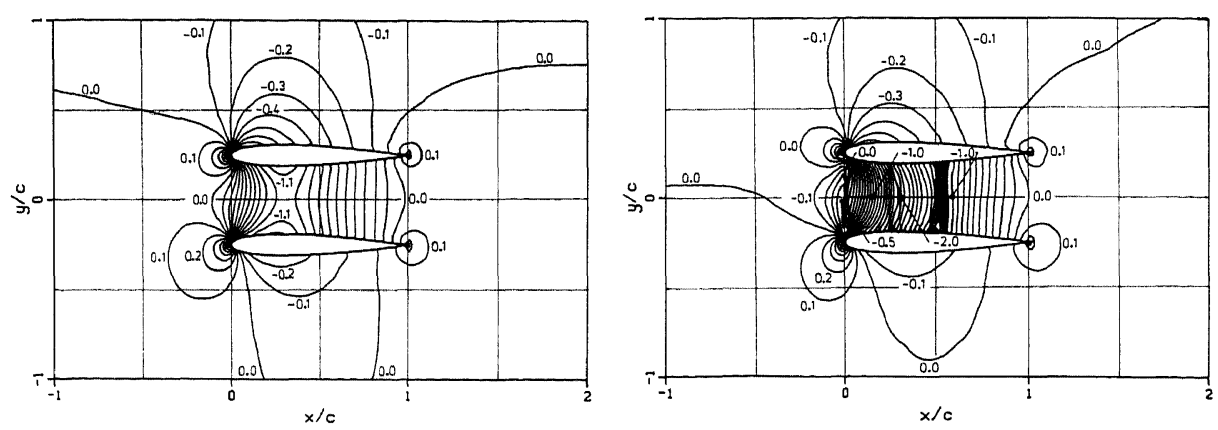

b. Pressure distributions $\left(c_{p}\right)$.
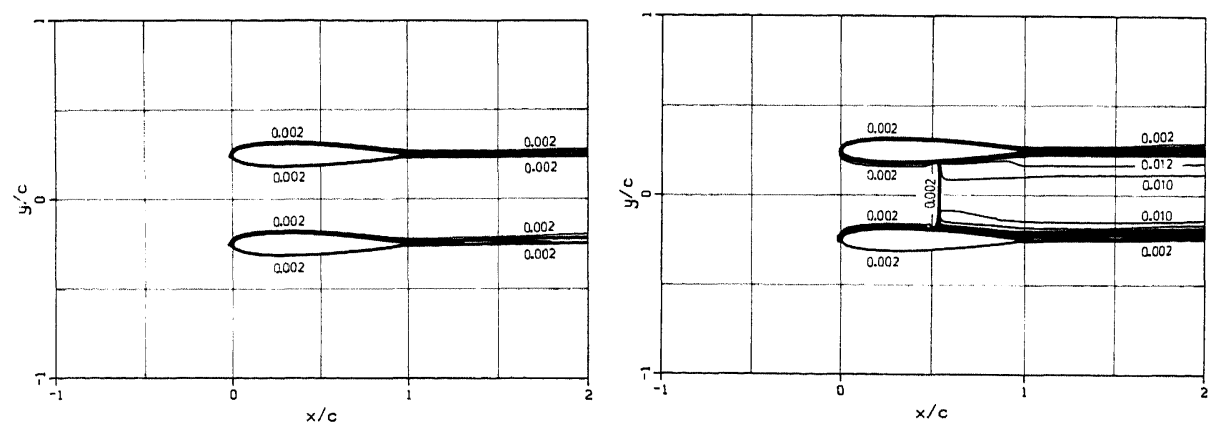

c. Entropy distributions $\left(s / s_{\infty}-1\right)$.

Fig. 4.6: Results for NACA0012-bi-airfoil with propeller disk, at $M_{\infty}=0.5, \alpha=2^{\circ}$, propeller off (left), propeller on (right). 
we have obtained a system of 6 equations with 6 unknowns $\left(\rho, u, v, p,\left(\delta_{2}\right)_{i, j}\right.$ and $\left.\left(\delta_{4}\right)_{i, j}\right)$ from which $\left(\delta_{2}\right)_{i, j}$ and $\left(\delta_{4}\right)_{i, j}$ can be computed. (So in each volume $\Omega_{i, j}$ which is intersected bv the propeller disk, the $x$-momentum and energy source are functions of $\delta$ and $q_{i, j}$ only.) In the IDeC-process(3.8) we take $r_{h}=r_{h}\left(q_{h}^{(n)}\right)$, with $\left(r_{h}\left(q_{h}^{(n)}\right)\right)_{i, j}=l_{i, j}\left(0,\left(\delta_{2}\right)_{i, j}^{(n)}, 0,\left(\delta_{4}\right)_{i, j}^{(n)}\right)^{T}$, where $l_{i, j}$ is the length of the propeller part inside $\Omega_{i, j}$. The use of source terms which depend on the solution fits perfectly well in the IDeCprocess.

For the case with working propeller we take $\delta=0.1$. For both cases (propeller on and off) we observe convergence to the solution of $F_{h}^{2}\left(q_{h}\right)=r_{h}\left(q_{h}\right)$. The values of lift and drag of the upper and lower airfoil (after the 5th IDeC-iteration) are given below.

Tab. 4.1: Lift and drag coefficients for NACA0012-bi-airfoil with propeller disk, at $M_{\infty}=0.5, \alpha=2^{\circ}$.

\begin{tabular}{|c|c|c|}
\hline & \multicolumn{2}{|c|}{ propeller disk } \\
\hline & off $(\delta=0)$ & on $(\delta=0.1)$ \\
\hline$c_{\text {iupper }}$ & -0.24 & -0.89 \\
\hline$c_{i \text { lower }}$ & 0.59 & 1.26 \\
\hline$c_{i i_{\text {upper }}}$ & -0.01 & 0.01 \\
\hline$c_{u_{\text {lower }}}$ & 0.02 & 0.06 \\
\hline
\end{tabular}

Clearly visible is the large influence of the working propeller on the lift force acting on each of the two airfoils.

In fig. 4.6 we give the Mach number, pressure and entropy distribution as obtained for both cases. In the Mach number distribution for the case with working propeller we clearly observe the Mach number increase and shock wave, which have been introduced in the internal flow part (and, consistently, the Mach number decrease in the external flow part). The imposed sources are such that the flow has developed from fully subsonic into transonic. In the pressure distribution (fig. 4.6b) for the case with.working propeller we observe that the assumed pressure jump appears indeed. Further, the corresponding entropy distribution (fig. 4.6c) shows the assumed isentropy through the propeller. For both cases (propeller on and off), the entropy which is spuriously generated at the airfoil noses and convected downstream, is an order of magnitude larger than for the single-airfoil. Cause of this is the non-smoothness of the grid at the noses of the bi-airfoil.

\section{CONCLUSIONS}

The Fromm scheme supplied with the Van Albada limiter yields second-order accurate solutions without spurious non-monotonicity and with sharp discontinuities. Comparison with the results of other investigators shows that for flows with discontinuities we obtain solutions of the same good quality on a grid which may be twice as course (in each coordinate direction).

For the computation of airfoil flows with the steady Euler equations, Iterative Defect Correction and nonlinear multigrid are found to be very efficient tools. It appears that it is sufficient to perform only a few IDeC-iterations; each implying only a single FAS-iteration.

An important property of the present method is that it is completely parameter-free; it needs no tuning of parameters.

\section{ACKNOWLEDGEMENTS}

The authors would like to thank J.W. Boerstoel and A. Kassies for providing O-type grids, P. Wesseling and P.W. Hemker for reading the manuscript, and H. Viviand and AGARD for permission to use reference results. 
RHFHENCES

1. G.D. van Albada, B. vali Leer ó W.W. Roberts (1982): A Comparative Study of Computational Methods in Cosmic Gasdynamics. Astron. Astrophys. 108, 76-84.

2. K. Böhmer, P.W. Hemker \& H.J. Stetrer (1984): The Defect Correction Approach. Computing, Suppl. 5, 1-32.

3. A. BRANDT (1982): Guide to Multigrid Development. Lecture Notes in Mathematics 960 , Springer Verlag.

4. S.K. Godunov (1959): Finite Difference Method for Numerical Computation of Discontinuous Solutions of the Equations of Fluid Dynamics (in Russian, also Cornell Aeronautical Lab. Transl.). Math. Sbornik 47, 272-306.

5. W. HACKBUSCH (1985): Multi-Grid Methods and Applications. Springer Verlag.

6. A. Harten, P. LaX \& B. van Leer (1983). On Upstream Differencing and Godunov-type Schemes for Hyperbolic Conservation Laws. SIAM Review 25, 35-61.

7. P.W. Hemker (1985): Defect Correction and Higher-Order Schemes for the Multigrid Solution of the Steady Euler Equations. Report NM-R8523, Centre for Mathematics and Computer Science, Amsterdam. To appear in Proceedings 2nd European Multigrid Conference, Cologne, 1985. Lecture Notes in Mathematics, Springer Verlag.

8. P.W. HEMKER \& B. KOREN (1986): A Non-linear Multigrid Method for the Steady Euler Equations. Report NM-R8621, Centre for Mathematics and Computer Science, Amsterdam. To appear in Proceedings GAMM-Workshop on The Numerical Simulation of Compressible Euler Flows, Rocquencourt, 1986. Vieweg Verlag Series Notes on Numerical Fluid Mechanics.

9. P.W. HEMKER \& S.P. SPEKREIJSE (1985): Multiple Grid and Osher's Scheme for the Efficient Solution of the Steady Euler Equations. Report NM-R8507, Centre for Mathematics and Computer Science, Amsterdam. To appear in Appl. Num. Math., 1986.

10. B. KOREN (1986): Euler Flow Solutions for a Transonic Windtunnel Section. Report NM-R8601, Centre for Mathematics and Computer Science, Amsterdam.

11. B. Koren (1986): Evaluation of Second Order Schemes and Defect Correction for the Multigrid Computation of Airfoil Flows with the Steady Euler Equations. Report NM-R8616, Centre for Mathematics and Computer Science, Amsterdam.

12. D. Küchemann (1978): The Aerodynamic Design of Aircraft. Pergamon Press.

13. B. VAN LeER (1982): Flux-Vector Splitting for the Euler Equations. Proceedings 8th International Conference on Numerical Methods in Fluid Dynamics, Aachen, 1982. Lecture Notes in Physics 170, Springer Verlag.

14. B. VAN LEER (1985): Upwind-Difference Methods for Aerodynamic Problems governed by the Euler Equations. Lectures in Applied Mathematics 22, AMS.

15. H.W. Liepmann \& A. RoshKo (1966): Elements of Gasdynamics. Wiley.

16. S. Osher \& F. Solomon (1982): Upwind Difference Schemes for Hyperbolic Systems of Conservation Laws. Math. Comp. 38, 339-374.

17. S. Osher \& S. Chakravarthy (1983): Upwind Schemes and Boundary Conditions with Applications to Euler Equations in General Geometries. J. Comp. Phys. 50, 447-481.

18. P.L. RoE (1981): Approximate Riemann Solvers, Parameter Vectors, and Difference Schemes. J. Comp. Phys. 43, 357-372.

19. S.P. SPEKREIJSE (1985): Second-Order Accurate Upwind Solutions of the $2 D$ Steady Euler Equations by the Use of a Defect Correction Method. Report NM-R8520, Centre for Mathematics and Computer Science, Amsterdam. To appear in Proceedings 2nd European Multigrid Conference, Cologne, 1985. Lecture Notes in Mathematics, Springer Verlag.

20. S.P. SPEKREIJSE (1986): Multigrid Solution of Monotone Second-Order Discretizations of Hyperbolic Conservation Laws. Report NM-R8611, Centre for Mathematics and Computer Science, Amsterdam. To appear in Math. Comp.

21. S.P. SPEKREISE (1986): A Comparison of Several Multigrid Methods for the Solution of SecondOrder Upwind Discretizations of the Steady Euler Equations. Report NM-R86xx, Centre for Mathematics and Computer Science, Amsterdam.

22. J.L. STEger \& R.F. WARming (1981): Flux-Vector Splitting of the Inviscid Gas Dynamic Equations with Applications to Finite-Difference Methods. J. Comp. Phys. 40, 263-293.

23. P.K. SwebY (1984): High Resolution Schemes using Flux Limiters for Hyperbolic Conservation Laws. SIAM J. Num. Anal. 21, 995-1011.

24. H. Viviand (1985): Numerical Solutions of Two-dimensional Reference Test Cases. In: Test Cases for Inviscid Flow Field Methods. H. YoshIHARA, et al. (eds.). AGARD Advisory Report 211. 